\title{
Ruthenium-Catalyzed Hydrogenation of Thioesters
}

\section{Key words}

ruthenium catalysis

hydrogenation

thioesters
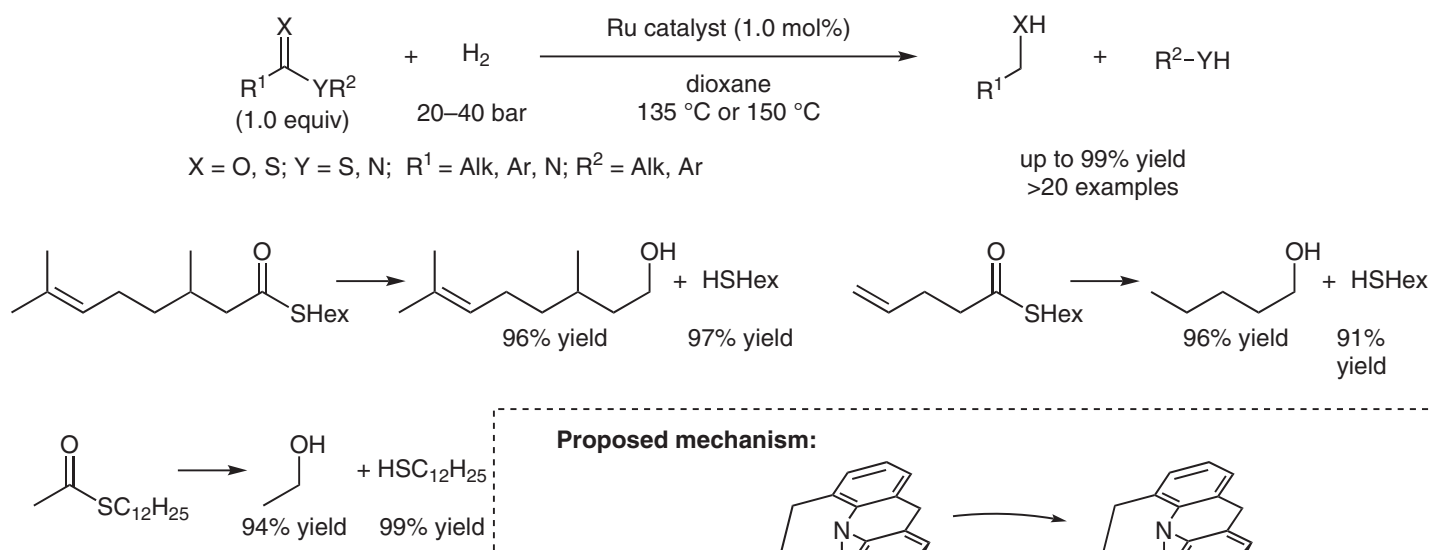

Proposed mechanism:
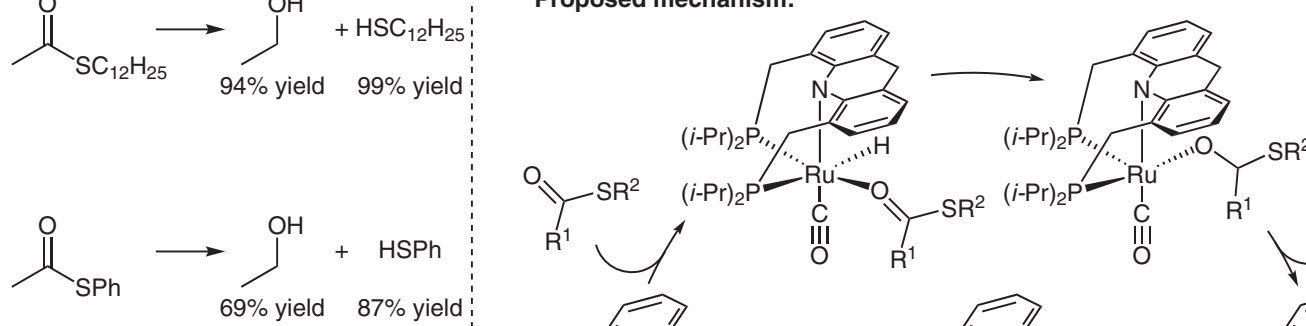

Significance: The authors report a rutheniumcatalyzed hydrogenation of thioesters, thiocarbamates and thioamides. The procedure provides waste-free access to alcohols, thiols and amines. An aciridine-based ruthenium complex is used as catalyst. The products are obtained in excellent yields. Whereas ketones and terminal alkenes are reduced, esters, amides, carboxylic acids and tertiary alkenes are tolerated by the hydrogenation protocol.
Comment: A plausible reaction mechanism based on mechanistic studies is proposed. Interestingly, the aldehyde is hydrogenated when used as a substrate in the presence of the thiol, suggesting an outer-sphere transition state. Other than the temperature, increasing the hydrogen pressure did not improve the yield significantly. It is assumed that heating facilitates the dissociation of the thiol from the ruthenium catalyst. 